For Internal Distribution Only

Accelerator Division

Alternating Gradient Synchrotron Department BROOKHAVEN NATIONAL LABORATORY

Upton, New York 11973

Accelerator Division

Technical Note

AGS/AD/Tech. Note No. 467

BNL ELECTRON BEAM TEST STAND

A. Pikin, J. Alessi, E. Beebe, A. Kponou, K. Prelec, L. Snydstrup

July 30, 1997 


\title{
BNL Electron Beam Test Stand
}

\author{
A. Pikin, J. Alessi, E. Beebe, A. Kponou, K. Prelec, L. Snydstrup \\ Brookhaven National Laboratory, Bldg. 930, Upton, NY 11973, USA
}

\begin{abstract}
The main purpose of the Electron Beam Test Stand (EBTS) Project at BNL is to build a versatile device to develop technologies that are relevant for a high intensity EBIS (Electron Beam Ion Source) and to study the physics of ion confinement in a trap. The EBTS will have all the main attributes of EBIS: a $1 \mathrm{~m}$ long, $5 \mathrm{~T}$ superconducting solenoid, electron gun, drift tube structure, electron collector, vacuum system, ion injection system, appropriate control and instrumentation. It therefore can be considered a short prototype of an EBIS for RHIC. The drift tube structure will be mounted in a vacuum tube inside a "warm" bore of a superconducting solenoid, it will be room temperature and its design will employ UHV technology to reach the $10^{-10}$ Torr level. The first gun to be tested will be a $10 \mathrm{~A}$ electron gun with high emission density and magnetic compression of the electron beam.
\end{abstract}

\section{Introduction}

The EBTS Project is part of a BNL program to build an EBIS for producing intense beams of highly charged ions of gold and uranium for subsequent acceleration in the RHIC accelerator complex (1). To meet the requirements of the accelerator, the intensity of ion beams should be $3 \cdot 10^{9}$ ions per pulse for $\mathrm{Au}^{35+}$ or $2 \cdot 10^{9}$ ions per pulse for $\mathrm{U}^{45+}$. If the output of the mentioned ions is $20 \%$ of the extracted total ion charge, and compensation of electrons by ions in a trap is $50 \%$, the space charge of electrons inside the ion trap (capacity of ion trap) should be $\mathrm{Q}_{\mathrm{el} .}=1.1 \cdot 10^{12}$ electrical charges. For an ion trap $1.5 \mathrm{~m}$ long, such a capacity corresponds to an electron beam with current $\mathrm{I}_{\mathrm{el} .}=10 \mathrm{~A}$ and energy $U_{\mathrm{el}}=20 \mathrm{kV}$. An electron beam with such parameters has not yet been used in EBISs, but has been used in other applications like powerful Magnicons and Klystrons, where the electron current is much higher (it is $250 \mathrm{~A}$ in Magnicon (2)). There are many inherent problems involved with a high intensity EBIS, such as: implementation of existing technology of electron beam formation (or developing a new one for the specific needs of EBIS), study of the ion losses in a high current electron beam, study of the efficiency of fast extraction and the effect of fast extraction and space charge on the emittance of the ion beam, development of the adequate technology of metal ion injection in a high capacity ion trap, development of a design and vacuum technology that can handle several tens of kilowatts of electron beam power with a background pressure in a region of ion trap $P_{\text {trap }} \approx 1 \cdot 10^{-10}$ Torr, and design of a fast control system. 
All the mentioned problems, and others that may come up in the process of performing experiments, will be studied on the Electron Beam Test Stand.

\section{Electron Beam Test Stand}

\subsection{General Construction of EBTS}

The construction of EBTS is determined by the goals of this project: to study experimentally specific problems of high intensity EBIS and, based on this experience, to develop the design and technology for building the RHIC EBIS. EBTS is planned to be a classical EBIS $(3,4)$ and will have all its main components: solenoid, electron gun, a system of insulated drift tubes, electron collector, ion extractor, gas and ion injection systems, a system of correcting coils for optimization of the magnetic field, if sensors, ion beam diagnostics (profile monitor, mass-spectrometer, emittance measuring system, energy spread measuring system). The preliminary assembly drawing of EBTS is presented in Fig. 1. The axis of EBTS will be positioned horizontally. The vacuum vessel of EBTS will consist of several (4-5) separate vacuum chambers with ConFlat flanges.

\section{$2.2 \quad$ Solenoid}

For electron beam focusing and confinement in a region of the ion trap, it is planned to use an unshielded superconducting solenoid with maximum magnetic field $5 \mathrm{~T}$ and "warm" bore. The requirement to modify axial distribution of magnetic field for various types of electron guns determined our choice of an unshielded solenoid. The longitudinal axis of the solenoid will be oriented horizontally. The suspension of the solenoid will be strong enough to withstand interaction with elements for shaping the magnetic field (iron shields and bucking coils). The length of the solenoid coil will be $1 \mathrm{~m}$ and the inner diameter of the "warm" bore will be $155 \mathrm{~mm}$. The period between refilling of the cryostat with liquid helium will be not less than 30 days in the persistent mode.

\subsection{Electron Beam and Space Charge Effect}

The maximum current of the electron beam inside the EBTS should be $10 \mathrm{~A}$. The energy of the electron beam in the ion trap region will be as low as possible and will be limited by the virtual cathode and stability of the electron beam. For electron current $I_{\mathrm{el}}=10 \mathrm{~A}$ and energy of the electron beam in the ion trap $E_{\mathrm{el}}=20 \mathrm{keV}$ (microperveance of ion beam $P_{\mu}=3.5$ ) the space charge of the electron beam in the volume of the ion trap with length $L_{\text {trap }}=0.6 \mathrm{~m}$ will be $Q_{1}=4.5 \cdot 10^{11}$ el.ch. If 
$D_{t} / D_{b}=16\left(D_{t}\right.$ - diameter of drift tube, $D_{b}$ - diameter of electron beam), the total radial potential well inside the drift tube will be $\Delta \mathrm{U}_{\mathrm{rad}}=7.1 \mathrm{kV}$. This is a substantial fraction of the electron energy. Possible ways to minimize the energy of the electron beam while maintaining stability can be by reducing the effect of the electron space charge by increasing the current of the electron beam during ion injection, so the electron space charge is considerably compensated, or ion injection into an initially high energy electron beam with subsequent deceleration.

In the case of a conventional electron gun with a straight electron beam, any reflected electrons are considered unwanted, because they affect operation of the electron gun and may have a harmful effect on the containment of ions in a trap. To reduce the fraction of electron beam reflected from regions with low potential (ion trap, electron collector), special care will be taken to have the transverse emittance of the electron beam as low as possible and employ the effects of a magnetic mirror. Reducing the emittance of the electron beam is also important for efficient retardation of the electron beam in the electron collector that is relevant for improving vacuum conditions and reducing the cost of power supplies and cooling system.

\section{$2.4 \quad$ Electron Guns}

The goal of the EBTS Project is to test different concepts of electron beam formation and different constructions of electron guns to achieve appropriate conditions for confinement, ionization and extraction of an ion beam with parameters required for RHIC. The first candidate to be tested may be a version with a semi-immersed gun with pure magnetic compression, based on a cathode with high emission density, capable of producing an electron beam up to $10 \mathrm{~A}$ and a current density of 200-300 A/ $\mathrm{cm}^{2}$ in the middle of the solenoid. The concept of a Brillouin gun is not ruled out and can also be tested, depending on the progress with experiments on the semi-immersed gun and simulations of a Brillouin gun.

\subsection{Drift Structure}

The system of drift tubes should allow us to have as low as possible radial potential and as low as possible ion losses during confinement in a trap. These requirements are contradictory, and the optimum value of $D_{\mathfrak{l}} / \mathrm{D}_{b}$ is to be found experimentally. The initial inner diameter of the drift tubes is planned to be $D_{t}=32 \mathrm{~mm}$. An electron beam diameter $D_{b}=2 \mathrm{~mm}$ gives us the ratio $D_{t} / D_{b}=16$.

Any sort of excitation of the electron beam is highly undesirable because it can cause heating of ions in a trap. One of the possible reasons for electron beam excitation can be a resonant 
interaction of the electron beam with the drift tube structure. To reduce this interaction, the inner diameter of the drift tubes and their length will be a little different from one tube to another. If high frequency instability of the electron beam still appears, if power absorbing lossy ceramic will be installed in the gaps between drift tubes. The average length of a regular drift tube will be $150 \mathrm{~mm}$; the ion trap will be confined within 4 such drift tubes located in a central part of superconducting solenoid.

\section{$2.6 \quad$ Electron Collector}

The construction of the electron collector should provide a temperature of the inner wall, when bombarded with electrons, that is well below the melting point of copper under a wide range of operating conditions and in all these ranges, the outgassing of the electron collector should be low enough for normal operation of the EBTS.

For cooling the electron collector, it is planned to use distilled water. The total inner area of the electron collector is $\sim 1000 \mathrm{~cm}^{2}$. For a time averaged power $P_{\text {aver }}=50 \mathrm{~kW}$ (power density is $\sim 0.05$ $\mathrm{kW} / \mathrm{cm}^{2}$ ), the calculated temperature difference between the cooling water and the wall of electron collector should be $\sim 30^{\circ} \mathrm{C}$. This means that this construction of the electron collector can handle local overheating with a power density more than 3 times higher than the averaged value, mentioned above.

The electron collector has bucking and transverse magnet correcting coils, a heater, and a thermocouple mounted in a separate chamber above the water cooled shield. All this assembly is installed inside an iron magnet shield and mounted on a high voltage $(40 \mathrm{kV})$ insulator in such a way that virtually only the entrance aperture in the electron collector connects the inner volume of the electron collector to the central part of EBTS. The construction of the electron collector and materials used for its manufacture allow baking it to $450^{\circ} \mathrm{C}$.

\section{$2.7 \quad$ Vacuum}

The vacuum system of EBTS has to provide a pressure of residual gas in the ion trap region $\mathrm{P} \approx 1 \cdot 10^{-10}$ Torr with an electron beam of $10 \mathrm{~A}$ running through it. This is a challenging requirement, considering the expected time averaged power dissipation in the electron collector is up to $50 \mathrm{~kW}$.

The drift tube structure will be installed inside the "warm" bore and will be bakeable to $300^{\circ} \mathrm{C}$. The concept of an EBIS with a "warm" structure of drift tubes is based on the existing technology of ultra high vacuum and on encouraging results at Saclay $(5,6)$ in experiments with a "warm" EBIS. 
This group obtained a pressure of residual (hydrogen) gas of $\mathrm{P}=5 \cdot 10^{-12}$ Torr in an EBIS vacuum chamber without an electron beam, and $\mathrm{P}=6 \cdot 10^{-10}$ Torr with an electron beam of $100 \mathrm{~mA}$. The concept of a "warm" EBIS has important advantages over a "cold" one: drift tubes do not condense gases and thus do not have a "memory effect", and all operations requiring exposure of EBIS to atmosphere can be done without warming up and cooling down the superconducting solenoid. The absence of a cryogenic vacuum separation of the ion trap from the gas injection region (that is typical for "cold" EBIS) is not so important if ion injection is used, as long as a good vacuum condition in the ion trap is provided. Nevertheless, gas injection is still considered mostly for controlled ion cooling. In this case, it will be done by direct continuous injection of cooling gas in a central part of the EBTS.

To satisfy the above-mentioned high vacuum requirements, it is planned to:

1. Reduce outgassing of all materials used in a central part by preliminary high temperature vacuum firing of almost all its components before installation, and by baking this part of the EBTS to $300^{\circ} \mathrm{C}$ after each exposure to atmosphere.

2. Reduce outgassing of the electron collector:

- by maximum reduction of the dissipated power of electron beam and by efficient cooling of the inner surface of the electron collector with water,

- by baking the electron collector to $450^{\circ} \mathrm{C}$ after each exposure to atmosphere and, if this will not be enough, by cleaning it with an ion discharge.

3. Restrict vacuum conductance between the central part, containing the ion trap, and heavily outgassing regions: the electron collector and electron gun, at the same time providing high pumping speed of these regions with separate vacuum pumps.

4. Provide the highest possible pumping speed for major components of residual gas, such as $\mathrm{H}_{2}, \mathrm{CO}, \mathrm{CH}_{4}$, in the central part.

The central part of the EBTS, containing the drift tube structure, will be pumped by the combination of titanium sublimation pump, cryopump, and high vacuum turbopump. This combination has total pumping speed $F_{\text {central }} \geq 2000 \mathrm{l} / \mathrm{sec}$ for room temperature hydrogen (the main expected component of residual gas). Estimated total outgassing rate of the central chambers, containing ionization region, is $\mathrm{Q}_{\text {outgas }}=1.3 \cdot 10^{-8} \mathrm{Tor} \cdot 1 / \mathrm{s}$. Gas flow from adjacent electron gun and electron collector chambers depends on pressure inside these chambers. The total vacuum conductivity of channels between central part and both mentioned electron gun and electron collector chambers is $\mathrm{S} \approx 84 \mathrm{l} / \mathrm{s}\left(\mathrm{H}_{2}, 300 \mathrm{~K}\right)$. If pressure of residual gas in these side chambers is $\mathrm{P}_{\text {side }}=2 \cdot 10^{-9}$ Tor, the estimated pressure in the central part is $\mathrm{P}_{\text {cent }} \approx 9 \cdot 10^{-11}$ Tor. 
Based on the outgassing rate of materials (stainless steel, alumina) and vacuum conductivity of the central part, the pressure drop inside central part between center and each side of the long central structure with drift tubes is estimated to be $\Delta \mathrm{P} \approx 6.7 \cdot 10^{-12}$ Tor.

\section{Ion Injection}

To provide injection of metal ions $(\mathrm{Au}, \mathrm{U})$ in the trap, EBTS will be equipped with an ion injection system, containing two external ion sources, mounted on different branches of a common beam line. Each line will contain lenses and electrostatic deflectors for focusing and steering of the ion beam. To switch injectors, it will be enough to switch polarity on a common electrostatic deflector. For gas injection and ion cooling it is planned to use a regular gas injection system with output in a central part of the EBTS. No vacuum separation for the gas injection is presently planned. As an additional option for metal injection, direct evaporation of metals by the electron beam in a region of ion trap is being considered.

\section{Diagnostics}

\subsection{RF Oscillations}

Apart from the routine current measurement on the drift tubes, we are planning to monitor If excitations in EBTS. To measure frequency and amplitude of excitations of the electron-ion system in the trap, it is planned to install UHV-compatible if sensors capable of picking up and transmitting signals, corresponding to different types of excitation, and ranging from $\sim 10 \mathrm{MHZ}$ (ion oscillations), to $\sim 20 \mathrm{GHz}$ (oscillations of electron beam, interacting with drift structure).

\subsection{Extracted Ion Beam}

\subsubsection{Charge State Spectrum}

Since for a high intensity EBIS with fast ion extraction the expected ion energy spread is broad, it is desirable to use for charge state and mass analysis a mass spectrometer with an energy focusing capability. The Mamyrin-type of time-of-flight mass spectrometer (TOF) (7), that employs an ion mirror to equalize the time of flight for ions with equal charge-to-mass ratio and different energy, can be considered as a good candidate for EBTS. To take advantage of energy focusing, it is planned to use a fast (10-20 ns) beam chopper. 


\subsubsection{Emittance Measuring Device}

For emittance measurements, it is planned to use an already existing device, based on the simultaneous measurement of ion beam distribution on a stack of strips after the narrow slit. This device is retractable and it will be installed in a chamber adjacent to the electron collector.

\subsubsection{Device for Measuring the Longitudinal Energy Spread}

For evaluation of the longitudinal energy spread of a full ion beam extracted from EBTS, it is planned to use a simple device with retarding mesh and an ion collector after the mesh. Special care will be taken to reduce the effect on the energy resolution of the incidence angle of the ion beam on the retarding mesh.

\section{Conclusion.}

Electron Beam Test Stand is in a stage of design. The concept of the "warm" bore of superconducting solenoid, with obvious advantage of cryogenic system, makes the task of maintaining high vacuum in ionization region, with high power electron beam running, more difficult, compare to concept with the "cold" bore. Nevertheless, preliminary calculations indicate, that with vacuum separation of heavily outgassing regions from ionization region and with appropriate vacuum technology it is possible to achieve and maintain the required vacuum. The architecture of the proposed EBTS is versatile enough to test different types of electron gun, drift tube structures, methods of electron beam formation and perform experiments with high current electron beams. 


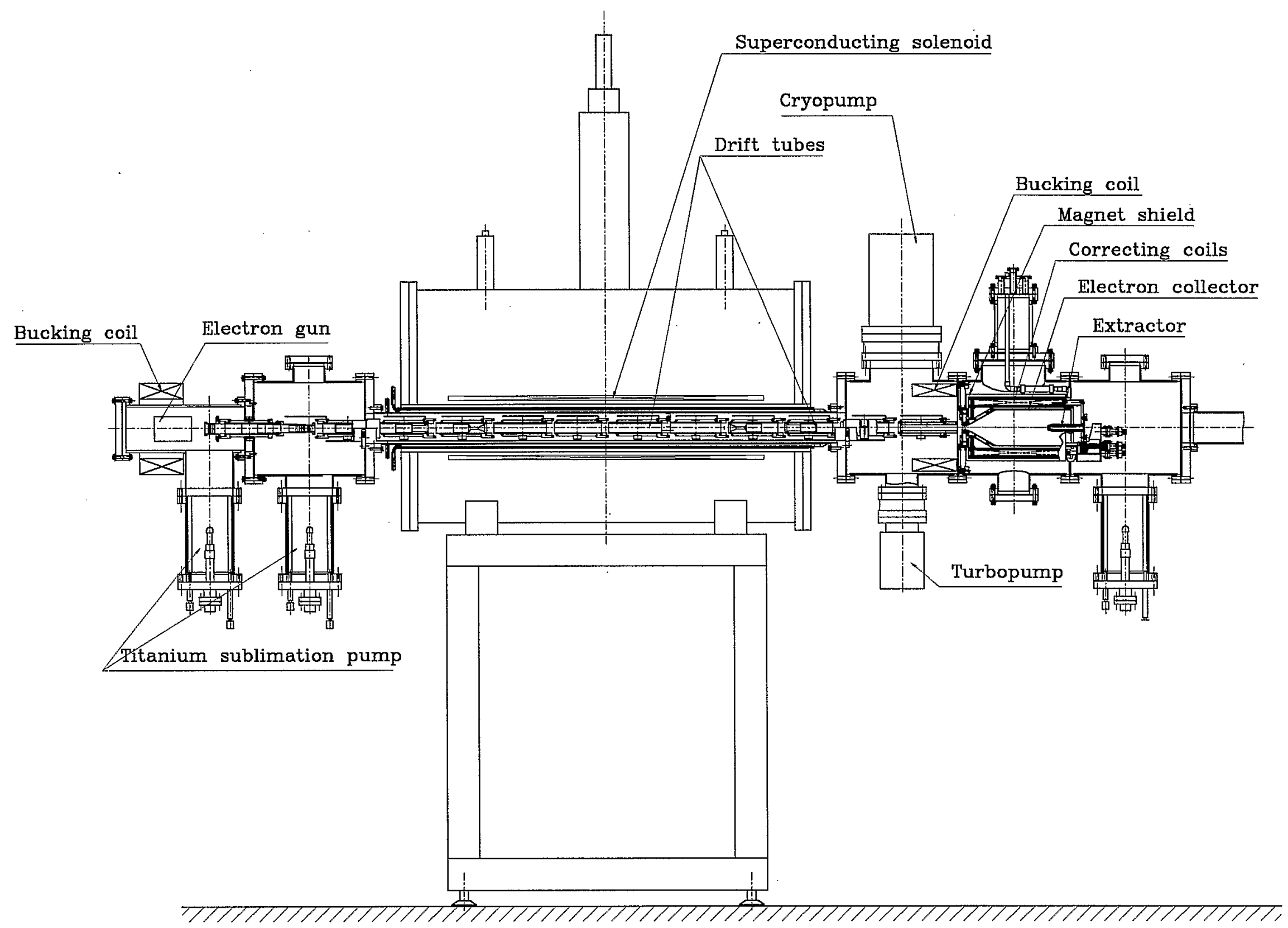

Fig. 1 BNL Electron Beam Test Stand 


\section{List of references.}

1. K. Prelec, J. Alessi, and A. Hershcovich BNL - 60554, European Particle Accelerator Conference - EPAC-94, London, England, June 27 - July 1, 1994.

2. Y.V. Baryshev, I.V Kazarezov,, E.V. Kozyrev, G.I. Kuznetsov, I.G. Makarov, O.A. Nezhevenko, B.Z. Persov, M.A. Tiunov, V.P. Yakovlev, I.A. Zapryagaev Nuclear Instrum. and Methods in Physics Research, A 340, (1994) 241 - 258.

3. E.D. Donets, USSR Inventor's Certificate No. 248860, 16 March 1967, Bull. OIPOTZ No. 23, 1969, P. 65.

4. E.D. Donets, V.I. Ilushchenko, V.A. Alpert

Proc. Premiere Conf. sur les Sources d'Ions, INSTM, Saclay, France, 1969, p. 625.

5. R. Gobin, S. Thuriez, G. Congretel, A. Courtois, J.P. Briand, O. Delferrière, J. Faure, G.

Giardino, F. Harrault, P. Leaux, P.A. Leroy, B. Visentin

Proc. 6-th International Conference on Ion Sources, September 10-16, 1995, Whistler, Canada.

6. G. Congretel, N. Rouvière and B. Visentin Vacuum, V. 45, No. 08-10, p. 777 - 780, 1995.

7. V.I.Karataev, B.A.Mamyrin,D.V.Shmikk, Zh. Tekh. Phiz. 41, 1971,1498. 